\title{
Universiteit
}

Leiden

The Netherlands

\section{Botulinum toxin for masseter hypertrophy}

Fedorowicz, Z.; Zuuren, E.J. van; Schoones, J.

\section{Citation}

Fedorowicz, Z., Zuuren, E. J. van, \& Schoones, J. (2013). Botulinum toxin for masseter hypertrophy. Cochrane Database Of Systematic Reviews, (9).

doi:10.1002/14651858.CD007510.pub3

Version: $\quad$ Not Applicable (or Unknown)

License: $\quad$ Leiden University Non-exclusive license

Downloaded from: https://hdl.handle.net/1887/100112

Note: To cite this publication please use the final published version (if applicable). 
Cochrane Database of Systematic Reviews

\section{Botulinum toxin for masseter hypertrophy (Review)}

Fedorowicz Z, van Zuuren EJ, Schoones J

Fedorowicz Z, van Zuuren EJ, Schoones J.

Botulinum toxin for masseter hypertrophy.

Cochrane Database of Systematic Reviews 2013, Issue 9. Art. No.: CD007510.

DOI: 10.1002/14651858.CD007510.pub3.

www.cochranelibrary.com 
TABLE OF CONTENTS

HEADER 1

ABSTRACT

PLAIN LANGUAGE SUMMARY

BACKGROUND

OBJECTIVES

METHODS

RESULTS

Figure 1.

DISCUSSION

AUTHORS' CONCLUSIONS

ACKNOWLEDGEMENTS

REFERENCES

CHARACTERISTICS OF STUDIES

APPENDICES

WHAT'S NEW

CONTRIBUTIONS OF AUTHORS

DECLARATIONS OF INTEREST

SOURCES OF SUPPORT

DIFFERENCES BETWEEN PROTOCOL AND REVIEW

INDEX TERMS

1

2

3

3

4

6

7

7

8

8

9

10

12

13

14

14

14

14 
[Intervention Review]

\section{Botulinum toxin for masseter hypertrophy}

Zbys Fedorowicz ${ }^{1}$, Esther J van Zuuren ${ }^{2}$, Jan Schoones ${ }^{3}$

1UKCC (Bahrain Branch), The Cochrane Collaboration, Awali, Bahrain. 2Department of Dermatology, Leiden University Medical Center, Leiden, Netherlands. ${ }^{3}$ Walaeus Library, Leiden University Medical Center, Leiden, Netherlands

Contact address: Zbys Fedorowicz, UKCC (Bahrain Branch), The Cochrane Collaboration, Box 25438, Awali, Bahrain. zbysfedorowicz@gmail.com.

Editorial group: Cochrane Movement Disorders Group.

Publication status and date: New search for studies and content updated (no change to conclusions), published in Issue 9, 2013.

Citation: Fedorowicz Z, van Zuuren EJ, Schoones J. Botulinum toxin for masseter hypertrophy. Cochrane Database of Systematic Reviews 2013, Issue 9. Art. No.: CD007510. DOI: 10.1002/14651858.CD007510.pub3.

Copyright @ 2013 The Cochrane Collaboration. Published by John Wiley \& Sons, Ltd.

\section{A B S T R A C T}

\section{Background}

Benign masseter muscle hypertrophy is an uncommon clinical phenomenon of uncertain aetiology which is characterised by a soft swelling near the angle of the mandible. The swelling may on occasion be associated with facial pain and can be prominent enough to be considered cosmetically disfiguring. Varying degrees of success have been reported for some of the treatment options for masseter hypertrophy, which range from simple pharmacotherapy to more invasive surgical reduction. Injection of botulinum toxin type A into the masseter muscle is generally considered a less invasive modality and has been advocated for cosmetic sculpting of the lower face. Botulinum toxin type $\mathrm{A}$ is a powerful neurotoxin which is produced by the anaerobic organism Clostridium botulinum and when injected into a muscle causes interference with the neurotransmitter mechanism producing selective paralysis and subsequent atrophy of the muscle.This review is an update of a previously published Cochrane review.

\section{Objectives}

To assess the efficacy and safety of botulinum toxin type A compared to placebo or no treatment, for the management of benign bilateral masseter hypertrophy.

\section{Search methods}

We searched the following databases from inception to April 2013: the Cochrane Central Register of Controlled Trials (CENTRAL); MEDLINE (via PubMed); EMBASE (via embase.com); Web of Science; CINAHL; Academic Search Premier (via EBSCOhost); ScienceDirect; LILACS (via BIREME); PubMed Central and Google Scholar (from 1700 to 19 April 2013). We searched two bibliographic databases of regional journals (IndMED and Iranmedex) which were expected to contain relevant trials. We also searched reference lists of relevant articles and contacted investigators to identify additional published and unpublished studies.

\section{Selection criteria}

Randomised controlled trials (RCTs) and controlled clinical trials (CCTs) comparing intra-masseteric injections of botulinum toxin versus placebo administered for cosmetic facial sculpting in individuals of any age with bilateral benign masseter hypertrophy, which had been self-evaluated and confirmed by clinical and radiological examination were considered for inclusion. We excluded participants with unilateral or compensatory contralateral masseter hypertrophy resulting from head and neck radiotherapy.

\section{Data collection and analysis}

Two review authors independently screened the search results. For future updates, two authors will independently extract data and assess trial quality using the Cochrane risk of bias tool. Risk ratios (RR) and corresponding $95 \%$ confidence intervals (CI) will be calculated for all dichotomous outcomes and the mean difference (MD) and $95 \% \mathrm{Cl}$ will be calculated for continuous outcomes. 


\section{Main results}

We retrieved 683 unique references to studies. After screening these references 660 were excluded for being non-applicable. We assessed 23 full text articles for eligibility and all of these studies were excluded from the review.

\section{Authors' conclusions}

We were unable to identify any RCTs or CCTs assessing the efficacy and safety of intra-masseteric injections of botulinum toxin for people with bilateral benign masseter hypertrophy. The absence of high level evidence for the effectiveness of this intervention emphasises the need for well-designed, adequately powered RCTs.

\section{PLAIN LANGUAGE SUMMARY}

\section{Botulinum toxin type A for masseter hypertrophy}

Masseter muscle hypertrophy occurs as a soft enlargement of the jaw muscles near the angle of the lower jaw and seldom presents a major health problem. However, in some individuals the swelling can be associated with pain or may be so large that it causes facial disfigurement. Although the cause of the condition is unclear it does appear to be more common in certain ethnic groups.

Symptoms such as pain can be treated with muscle relaxants and may also include bite adjustments or involve the use of splints on the teeth. Surgical reduction of the jaw muscle or injections of botulinum toxin type A directly into the muscle are other treatment options. Botulinum toxin type $A$ is a powerful neurotoxin produced by the anaerobic organism Clostridium botulinum. When botulinum toxin type $A$ is injected into a muscle it causes interference with the neurotransmitter mechanism producing selective loss of muscle function and a subsequent decrease in the mass of the muscle.

Although the use of botulinum toxin injections might appear to have certain advantages over surgery the authors of this review did not find any high quality studies that evaluated the effectiveness and potential side effects of botulinum toxin type A for the management of benign masseter hypertrophy. Well-designed randomised controlled trials are needed to assess the effectiveness and safety (i.e. side effects) of this intervention. 


\section{B A C K G R O U N D}

\section{Aetiology and prevalence}

Benign masseter muscle hypertrophy is an uncommon clinical phenomenon of uncertain aetiology. It is characterised by a soft swelling, near the angle of the mandible, which can be associated with facial pain. The hypertrophy can be prominent enough to be considered cosmetically disfiguring.

More than 250 cases of benign bilateral masseter muscle hypertrophy have been reported since its first published description (Legg 1880). Prevalence data are scarce but in a recent study (Sannomya 2006) 90 (4\%) of the patients with masseter hypertrophy were less than 10 years old and 3\% were over 40 years of age (mean 30 years), with a male to female ratio of 1:1.

The aetiology of masseter muscle hypertrophy has been attributed to a number of factors including: emotional stress, chronic bruxism, masseteric hyper-function and para-function, and microtrauma (Harriman 1996; Serrat 1998; Wilson 1990). It reportedly occurs most frequently among pacific Asians and is associated with ethnic characteristics (e.g. prominence of the mandibular angle) and dietary habits (Jin Park 2007). The findings of several investigators suggest that the increase in muscle size is not caused by work hypertrophy but as a result of compensatory enlargement due to lack of a certain type of muscle fibre (Jin Park 2007; Satoh 2001). Tests have shown that the composition of muscle fibres in the enlarged masseter is very different from that in muscles with 'work hypertrophy' as well as that in normal masseter muscles (Satoh 2001), suggesting that the term 'hypertrophy' could be potentially misleading.

Other possible causes and associations have been suggested including: clenbuterol induced hypertrophy, overuse of anabolic steroids (Skoura 2001), localised scleroderma and facial hemiatrophy (Kim 2000), and a multifactorial origin in combination with a genetic basis (Giudice 1992). Benign masseter hypertrophy is also compatible with a rare genetic condition known as hypertrophic branchial myopathy (Kitagawa 2000).

\section{Description of the condition}

\section{Signs and symptoms}

Bilateral enlargement of the masseter muscles is often accompanied by pain, which may be intermittent and can be confused with pain arising from the parotid gland (Newton 1999; Nishida 1995). Clinical examination usually reveals a soft tissue mass near the angle of the mandible, which becomes more prominent on clenching of the teeth (Sannomya 2006).

Limitation of the mouth opening has been reported in some cases and particularly where the muscles are focally dystonic with tension in the region of the hypertrophied muscle (Papapetropoulos 2006). Midline deviation has also been observed in some cases, as well as masseteric (hemi-masticatory) spasm (Kim 2000). It has also been suggested that the hypertrophied muscles of the jaw can lead to increased pressure in the temporomandibular joints (TMJ), which can generate severe pain and mimic temporo-mandibular dysfunction syndrome (TMD) (Chikani 2003).
Diagnosis cannot solely be based on clinical findings and there are conflicting recommendations in the literature for investigating patients presenting with benign bilateral masseter hypertrophy. These recommendations include the following.

- Standard radiographs (not essential but can sometimes assist in diagnosis).

- Computed tomographic (CT) scan, magnetic resonance imaging (MRI) scan, or both (considered the gold standard in confirming a clinical suspicion).

- Muscle biopsy.

- Morphometric analysis.

- Ultrasonographic measurement.

- Electromyographic measurement.

\section{Description of the intervention}

\section{Treatment options}

A range of treatment modalities have been reported with variable degrees of success and failure. Treatment options include the following.

1. Pharmacotherapy: anxiolytics, muscle relaxants and antidepressants.

2. Dental restorations and occlusal adjustments to correct premature contacts and malocclusions, and prevention of parafunctional habits with orthotic appliances.

3. Botulinum toxin type $A$ injections into the muscle.

4. Radiofrequency volumetric reduction.

5. Intra-oral and extra-oral surgical reduction of masseter size, removal of mandibular angle, neurectomy of the masseteric nerve, and resection of the buccal fat pad.

\section{How the intervention might work}

Botulinum toxin type $A(B t A)$ is a powerful neurotoxin produced by the anaerobic organism Clostridium botulinum. When BtA is injected into a muscle it causes interference with the neurotransmitter mechanism producing selective paralysis and subsequent atrophy of the muscle.

\section{Why it is important to do this review}

Surgery has historically been the standard treatment for cosmetic reduction of masseter hypertrophy, but injection of BtA into the muscle, which is generally considered to be a less invasive modality, has more recently been advocated. This systematic review is an update of a previously published Cochrane review (Al-Muharraqi 2009).

\section{O B JECTIVES}

To assess the efficacy and safety of botulinum toxin type A compared to placebo or no treatment, for the management of benign bilateral masseter hypertrophy.

\section{Diagnosis}




\section{METHODS}

\section{Criteria for considering studies for this review \\ Types of studies}

Randomised controlled trials (RCTs) and controlled clinical trials (CCTs) were considered for inclusion.

\section{Types of participants}

Individuals of any age with bilateral benign masseter hypertrophy which has been self-evaluated and confirmed by clinical and radiological examination were considered for inclusion. In view of the possible clinical diversity in presentation, we excluded studies involving participants with unilateral or compensatory contra lateral masseter hypertrophy resulting from head and neck radiotherapy from this review.

\section{Types of interventions}

Interventions included transcutaneous intra-masseteric injections of botulinum toxin versus placebo or no treatment. We sought to include studies in which the intervention had been administered for cosmetic facial sculpting. We considered studies involving a single injection cycle in addition to studies in which all participants entered in to a trial had received repeat injections at similar time periods.

\section{Types of outcome measures}

Assessment was to include a follow-up period of up to two years after the intervention.

\section{Primary outcomes}

1. Self-assessed improvement in facial appearance and patient satisfaction using any validated scale or questionnaire.

2. Patient-assessed improvement in pain or discomfort associated with the temporo-mandibular joints or jaw muscles using any recognised validated pain scale.

\section{Secondary outcomes}

1. Objective evaluation of the change in facial contour, involving physical measurement. Change in facial contour could be measured by clinical photography or radiological measurement which could include three-dimensional computed tomographic (CT) scans, magnetic resonance (MR) imaging, or ultrasonographic measurements of the thickness of the masseter muscle.

2. Adverse events including any specific adverse effects, systemic or local toxicity, any clinically diagnosed hypersensitivity and other unacceptable events associated with this treatment.

\section{Search methods for identification of studies}

\section{Electronic searches}

\section{Databases searched}

We extended and updated the searches for the following databases:

- MEDLINE (via Pubmed) (1950 to 19 April 2013). See Appendix 1

- EMBASE (via embase.com) (from 1980 to 19 April 2013). See Appendix 2

- Web of Science (from 1945 to 19 April 2013). See Appendix 3
- the Cochrane Central Register of Controlled Trials (CENTRAL) (The Cochrane Library 2013, Issue 4). See Appendix 4

- CINAHL (from 1982 to 19 April 2013). See Appendix 5

- Academic Search Premier (via EBSCOhost) (from 1897 to 19 April 2013). See Appendix 6

- ScienceDirect (from 1823 to 19 April 2013). See Appendix 7

- LILACS (via BIREME) (from 1982 to 19 April 2013). See Appendix 8

- PubMed Central (from 1809 to 19 April 2013). See Appendix 9

- Google Scholar (from 1700 to 19 April 2013). See Appendix 10

Although we did not search the Cochrane Movement Disorders Group Trials Register we clarified that it did not contain any relevant studies.

We updated our search of IndMED, a bibliographic database of Indian journals, available at (http://indmed.nic.in/) and a similar Iranian database, Iranmedex, available at (www.iranmedex.com), using free text terms appropriate for this review on 27 April 2013.

\section{Searching other resources}

We did not conduct any handsearching of journals but searched the reference lists of relevant articles in addition to the review authors' personal database of trial reports. We also contacted a number of investigators by electronic mail to ask for details of additional published and unpublished trials. There were no language restrictions on included studies and we arranged to translate any relevant non-English papers.

\section{Data collection and analysis}

\section{Selection of studies}

Two review authors (ZF and EvZ) independently assessed the abstracts of studies resulting from the searches. We obtained full copies of all relevant and potentially relevant studies, those appearing to meet the inclusion criteria, and those for which there were insufficient data in the title and abstract to make a clear decision.The two review authors independently assessed the full text papers and resolved any disagreement on the eligibility of included studies through discussion and consensus. We excluded all irrelevant records and noted details of the studies and the reasons for their exclusion in the 'Characteristics of excluded studies' table in RevMan 5.2 (RevMan 2012).

\section{Data extraction and management}

The following methods of data extraction and management will apply for subsequent updates, and when future studies are identified. We will enter study details into the 'Characteristics of included studies' table in RevMan 5.2. The review authors will collect outcome data using a pre-determined form designed for this purpose. Two authors (ZF and EvZ) will enter extracted data into RevMan 5.2., data will only be included if there is an independently reached consensus.

We will extract the following details.

1. Trial methods: (a) method of allocation, (b) masking of participants, personnel and outcome assessors, (c) exclusion of participants after randomisation and proportion and reasons for losses at follow-up. 
2. Participants: (a) country of origin and location (i.e. private clinic or academic institute), (b) sample size, (c) age, (d) sex, (e) inclusion and exclusion criteria.

3. Intervention: (a) type, dosage, route of administration, (b) length of follow-up.

4. Control: (a) type, dosage, route of administration, (b) length of follow-up.

5. Outcomes: (a) primary and secondary outcomes pre-specified in the 'Types of outcome measures' section of this review.

If stated, we will record the sources of funding of any of the included studies.

\section{Assessment of risk of bias in included studies}

If studies are included in future updates each review author will grade the selected trials and assess every trial using a simple contingency form following the domain-based evaluation described in the Cochrane Handbook for Systematic Reviews of Interventions 5.1.0 (Higgins 2011). We will compare the evaluations and discuss and resolve any inconsistencies and disagreements.

The following domains will be rated separately for each of the included studies as 'low risk of bias', 'high risk of bias', and 'unclear' if the risk of bias was uncertain or unknown:

1. sequence generation;

2. allocation concealment;

3. blinding (of participants, personnel and outcome assessors);

4. incomplete outcome data;

5. selective outcome reporting;

6. other bias.

These assessments will be reported in the 'Risk of bias' table for each individual study in the 'Characteristics of included studies' section of the review.

We will also categorise and report the overall risk of bias of each of the included studies according to the following:

- low risk of bias (plausible bias unlikely to seriously alter the results) if all criteria were met;

- unclear risk of bias (plausible bias that raises some doubt about the results) if one or more criteria were assessed as unclear; or

- high risk of bias (plausible bias that seriously weakens confidence in the results) if one or more criteria were not met.

These assessments will be reported in the 'Risk of bias in included studies' section of this review.

\section{Measures of treatment effect}

If studies are included in future updates we will conduct analysis at the same level as the allocation. We will calculate risk ratios (RR) and corresponding $95 \%$ confidence interval $(\mathrm{Cl})$ for all dichotomous outcomes and the mean difference (MD) and $95 \% \mathrm{Cl}$ for continuous outcomes. We will use RevMan 5 for data analysis. Unless stated otherwise, we will use the Mantel-Haenzel method to calculate the RR for dichotomous outcomes and MD for continuous outcomes. As it is likely that the timing of outcome assessment will vary between studies we will consider grouping the data according to the following time-points: six months, one year and two years.

\section{Unit of analysis issues}

We expect to include trials of participants with bilateral hypertrophy in which the masseter muscles of an individual participant were the units of randomisation and subsequent analysis. We will analyse these data based on the advice provided in sections 9.3.8 in the Cochrane Handbook for Systematic Reviews of Interventions (Higgins 2011).

\section{Dealing with missing data}

If studies are included in future updates we will make attempts to retrieve missing data from the investigators of the included trials, and if unsuccessful or the discrepancies are significant, we will provide a narrative synthesis of the data as reported.

\section{Assessment of heterogeneity}

If studies are included in future updates we will assess clinical heterogeneity by examining the characteristics of the studies and the similarity between types of participants, interventions and outcomes. In view of the expectation of a degree of clinical heterogeneity between the studies we will use a random-effects model for statistical analyses. Statistical heterogeneity will be assessed using the $\mathrm{Chi}^{2}$ test and the $\mathrm{I}^{2}$ statistic, where $\mathrm{I}^{2}$ values over $50 \%$ indicate moderate to high heterogeneity (Higgins 2011).

\section{Assessment of reporting biases}

if future updates include studies we will follow the recommendations on testing for funnel plot asymmetry as described in section 10.4.3.1 of the Cochrane Handbook for Systematic Reviews of Interventions 5.1.0 (Higgins 2011) to assess publication bias.

\section{Data synthesis}

If future updates include studies two review authors (ZF and EvZ) will analyse the data and report the results as specified in Chapter 9 of the Cochrane Handbook for Systematic Reviews of Interventions Version 5.1.0 (Higgins 2011). Where appropriate, we will calculate the pooled $\mathrm{RR}$ and $95 \% \mathrm{Cl}$ for dichotomous outcomes and the pooled MD and $95 \% \mathrm{Cl}$ for continuous outcomes. We will use the Mantel-Haenzel method for combining results across studies using random-effects models. In the event that there are insufficient clinically homogeneous trials for any specific intervention or insufficient study data that can be pooled, we will present a narrative synthesis.

\section{Subgroup analysis and investigation of heterogeneity}

If studies are included in future updates we will perform subgroup analysis by dose (i.e. low dose of BtA compared to medium or high doses). We define a low dose of BtA as $\leq 150 \mathrm{U}$ per muscle and a medium to high dose as $>150 \mathrm{U}$ per muscle.

\section{Sensitivity analysis}

If future updates contain a sufficient number of included studies we plan to conduct sensitivity analyses to assess the robustness of our review results. We will do this by repeating the analyses excluding studies with unclear or inadequate allocation concealment, blinding of outcomes assessment and completeness of follow-up. 


\section{RESULTS}

\section{Description of studies}

\section{Results of the search}

A literature search conducted on April 19, 2013 identified 1147 records. After duplicates were removed, a total of 683 references remained for review of titles and abstracts (PubMed 258, EMBASE 361 (34 abstracts to conference proceedings), from which 181 unique, Web of Science: 181 (8 abstracts to conference proceedings), from which 41 unique, CENTRAL 105, from which 97 unique, CINAHL 23, from which 3 unique, Academic Search Premier 55, from which 11 unique, ScienceDirect: 47, from which 10 unique, LILACS: 5, from which 3 unique, PubMed Central/ PMC: 54, from which 48 unique, Google Scholar: unknown set, 13 unique), citations to Cochrane Review: through Web of Science: 2, from which 1 unique, citations from Cochrane Review: through Web of Science: 30, from which 11 unique and citations to Cochrane Review: through Google Scholar: 13, from which 6 unique). After examination of the titles and abstracts of these references, we eliminated all but 23 and excluded them from further review (see Figure 1). We obtained full text copies of those remaining studies, translated them into the English language as required and subjected them to further evaluation. We examined the bibliographical references of these studies and, as with our searches of the IndMED and Iranmedex databases, they did not provide any further citations to potentially eligible studies. Two authors, Zbys Fedorowicz (ZF) and Esther van Zuuren (EvZ), independently assessed all of the full text papers, and resolved any disagreement on their eligibility for this review through discussion and consensus. 
Figure 1. Study flow diagram.

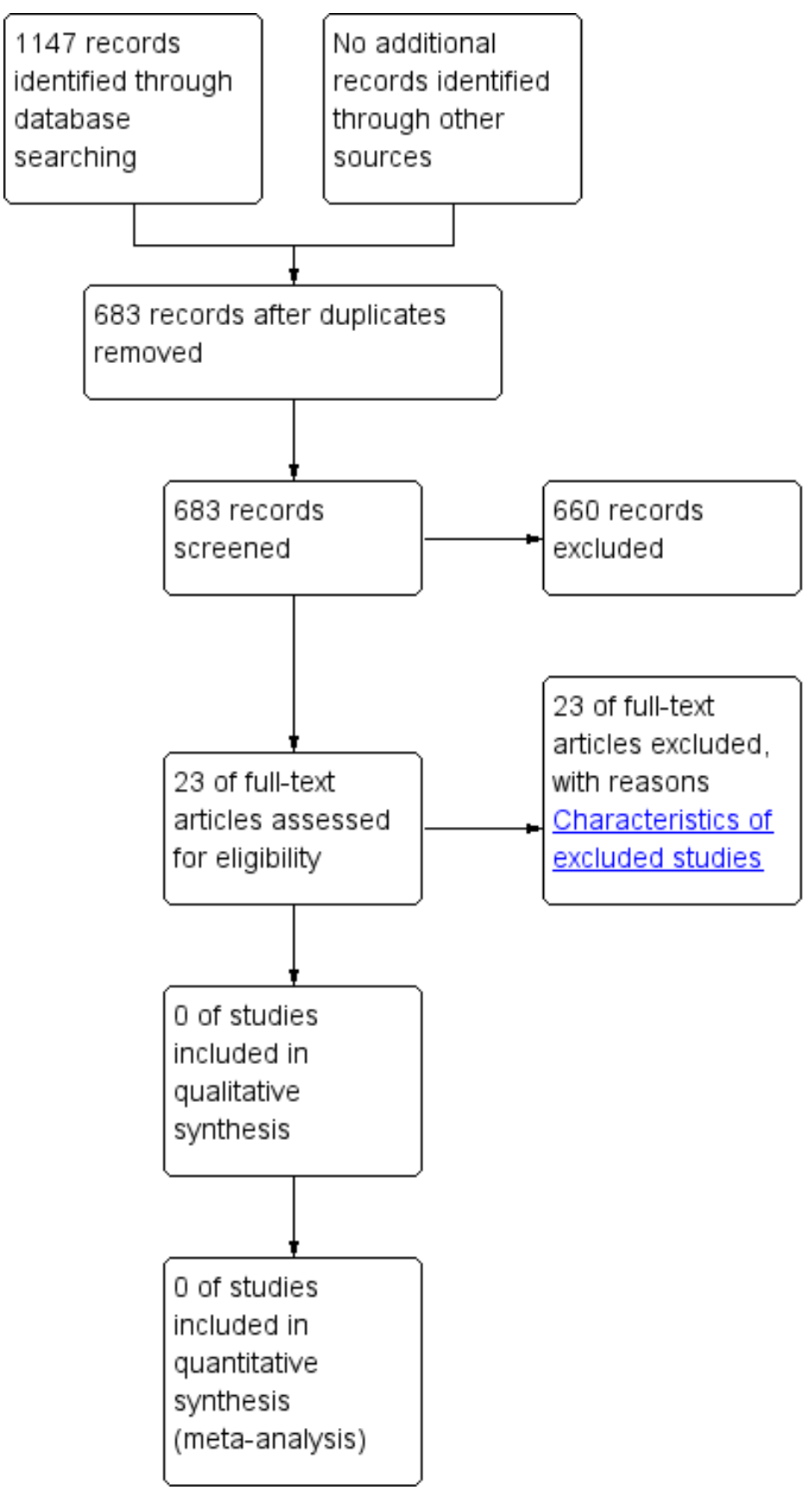

\section{Included studies}

We retrieved a number of studies in our comprehensive search of the literature but none were eligible and therefore no trials were included in this review.

\section{Excluded studies}

We excluded all records which did not match our inclusion criteria and noted the reasons for their exclusion in the 'Characteristics of excluded studies' table.

\section{Risk of bias in included studies}

A risk of bias analysis was not carried out because no trials were included in the review.

\section{Effects of interventions}

None of the studies retrieved in our searches met our inclusion criteria and therefore no data were available for analysis.

\section{DISCUSSION}

The comprehensive search used in this review provided a large number of references to trials and thus the lack of relevant 
randomised controlled trials or controlled clinical trials as well as any robust evidence to support or refute the effectiveness of botulinum toxin for masseter hypertrophy, proved to be somewhat disappointing. Over the last 10 years a number of case reports and several recent cohort studies have sought to illustrate the effectiveness of botulinum toxin type $A$ injections for benign masseter muscle hypertrophy, but questions remain unanswered as to whether management options based on this intervention can be considered both effective and safe.

\section{AUTHORS' CONCLUSIONS}

\section{Implications for practice}

A lack of consensus on the aetiology of benign masseter hypertrophy together with an increasing concern about the longterm effects of botulinum toxin type $\mathrm{A}$ (BtA) would appear to underline the fact that its therapeutic benefits remain unclear. Therefore, before selecting this treatment option clinicians need to carefully consider, and indeed discuss, not only its benefits but also the possibility and implications of any potential harms with their patients.

\section{Implications for research}

This systematic review on botulinum toxin for masseter hypertrophy did not identify any eligible studies. This review highlights the need for well-designed, adequately powered randomised controlled trials to evaluate the efficacy and safety of botulinum toxin for reducing the size and volume of the masseter muscles in people diagnosed with bilateral benign masseter hypertrophy. Although further research is required, conducting randomised controlled trials for this intervention will present challenges in terms of the willingness of participants to be enrolled into a trial where they may be allocated to an intervention which will result in unilateral facial deformity for the duration of the trial. Outcomes assessments in future trials should aim to include both subjective and objective baseline and post treatment evaluations, such as maximum bite force, clinical photography, physical measurements of changes in facial contour, cephalometry and electromyographic studies of masseter function. Any future trials will also need to be rigorous in design and delivery, with subsequent reporting to include high quality descriptions of all aspects of methodology to enable appraisal and interpretation of results, and conform with the Consolidated Standards of Reporting Trials (CONSORT) statement (http://www.consortstatement.org/).

\section{ACK N O WLEDGEMENTS}

The review authors would like to acknowledge the assistance they have received from members of the Cochrane Movement Disorders Group and for the helpful comments on this review from the referees. We would like to thank Emma Low of GKT Dental Institute, King's College Hospital University of London, who provided us with full text copies of many of the papers and Raphael F. de Souza of the Department of Dental Materials and Prosthodontics, Ribeirão Preto Dental School, University of São Paulo, Brazil who very kindly ran all the searches for the original version of this review. The authors would like to acknowledge the contribution of Mohammed Al Muharraqi, Jaffer Al Bareeq, Reem Al Bareeq, and Mona Nasser to earlier versions of this review. 


\section{RE F E R E N C E S}

\section{References to studies excluded from this review}

Ahn 2007 \{published data only\}

Ahn KY, Kim ST. The change of maximum bite force after botulinum toxin type a injection for treating masseteric hypertrophy. Plastic and Reconstructive Surgery 2007;120(6):1662-6.

\section{Bhogal 2006 \{published data only\}}

Bhogal PS, Hutton A, Monaghan A. A review of the current uses of Botox for dentally-related procedures. Dental Update 2006;33(3):165-8.

\section{Castro 2005 \{published data only\}}

Castro WH, Gomez RS, Da Silva Oliveira J, Moura MD, Gomez RS. Botulinum toxin type $A$ in the management of masseter muscle hypertrophy. Journal of Oral and Maxillofacial Surgery 2005;63(1):20-4

\section{Chikani 2003 \{published data only\}}

Chikhani L, Dichamp J. Bruxism, temporo-mandibular dysfunction and botulinum toxin. Annales de Réadaptation et de Médecine Physique 2003;46(6):333-7.

\section{Choe 2005 \{published data only\}}

Choe SW, Cho WI, Lee CK, Seo SJ. Effects of botulinum toxin type A on contouring of the lower face. Dermatologic Surgery 2005;31(5):502-7.

\section{Collier 2009 \{published data only\}}

Collier J, Zou L. Quantitative measurement of the response of masseteric hypertrophy to treatment with botulinum toxin type-A. British Journal of Oral and Maxillofacial Surgery 2009;47(7):e30.

\section{Hui 2002 \{published data only\}}

Hui AC. Botulinum toxin for treatment of masseteric hypertrophy. Journal of Neurology 2002;249(3):345.

\section{Ișeri 2004 \{published data only\}}

Işeri M, Işeri PK. Treatment of unilateral masseter hypertrophy with botulinum toxin in two cases. Kulak Burun Boğaz Ihtisas Dergisi 2004;12(3-4):99-102.

Jin 2010 \{published data only\}

Jin SP, Cho S, Youn C, Feneran A, Haw S, Bak H, et al. Effectiveness of botulinum toxin type $A$ and $B$ in benign masseteric hypertrophy. Journal of Dermatology 2010;37(Suppl 1):78.

\section{Kane 2007 \{published data only\}}

Kane MA. Botulinum toxin A for lower facial contouring: A prospective study. Aesthetic Plastic Surgery 2007;31(5):452-3.

\section{Kaynar 1997 \{published data only\}}

Kaynar A, Parman Y, Poyanl A, Demirkol O, Birinci SH, Minareci $O$, et al. Therapeutic use of botulinum toxin in the treatment of masseter hypertrophy. International Journal of Oral and Maxillofacial Surgery 1997;26(Suppl 1):116.
Kim 2003 \{published data only\}

Kim HJ, Yum KW, Lee SS, Heo MS, Seo K. Effects of botulinum toxin type $A$ on bilateral masseteric hypertrophy evaluated with computed tomographic measurement. Dermatologic Surgery 2003;29(5):484-9.

Lee 2007 \{published data only\}

Lee CJ, Kim SG, Kim YJ, Han JY, Choi SH, Lee SI. Electrophysiologic change and facial contour following botulinum toxin A. Plastic and Reconstructive Surgery 2007;120(3):769-78.

\section{Mischkowski 2005 \{published data only\}}

Mischkowski RA, Siessegger M, Lazar F, Zöller JE. Chemodenervation with botulinum toxin in masseteric hypertrophy. Mund-, Kiefer- und Gesichtschirurgie 2005;9(2):101-8.

\section{Moore 1994 \{published data only\}}

Moore AP, Wood GD. The medical management of masseteric hypertrophy with botulinum toxin type A. British Journal of Oral and Maxillofacial Surgery 1994;32(1):26-8.

Niamtu 1999 \{published data only\}

Niamtu J. Aesthetic uses of botulinum toxin A. Journal of Oral and Maxillofacial Surgery 1999;57(10):1228-33.

\section{Park 2003 \{published data only\}}

Park MY, Ahn KY, Jung DS. Botulinum toxin type A treatment for contouring of the lower face. Dermatologic Surgery 2003;29(5):477-83.

Rogers 1995 \{published data only\}

Rogers BA, Whear NM. Medical management of masseteric hypertrophy. Journal of Oral and Maxillofacial Surgery 1995;53(4):492.

\section{Smyth 1994 \{published data only\}}

Smyth AG. Botulinum toxin treatment of bilateral masseteric hypertrophy. British Journal of Oral and Maxillofacial Surgery 1994;32(1):29-33.

\section{To 2001 \{published data only\}}

To EW, Ahuja AT, Ho WS, King WW, Wong WK, Pang PC, et al. A prospective study of the effect of botulinum toxin $A$ on masseteric muscle hypertrophy with ultrasonographic and electromyographic measurement. British Journal of Plastic Surgery 2001;54(3):197-200.

\section{von Lindern 2001 \{published data only\}}

von Lindern JJ, Niederhagen B, Appel T, Bergé S, Reich RH. Type A botulinum toxin for the treatment of hypertrophy of the masseter and temporal muscles: an alternative treatment. Plastic and Reconstructive Surgery 2001;107(2):327-32.

von Lindern 2003 \{published data only\} von Lindern JJ, Niederhagen B, Bergé S, Appel T. Type A botulinum toxin in the treatment of chronic facial pain 
associated with masticatory hyperactivity. Journal of Oral and Maxillofacial Surgery 2003;61(7):774-8.

Yu 2007 \{published data only\}

Yu CC, Chen PK, Chen YR. Botulinum toxin a for lower facial contouring: a prospective study. Aesthetic Plastic Surgery 2007;31(5):445-51.

\section{Additional references}

\section{Giudice 1992}

Giudice M, Marra A, Barba A, Passariello N, D'Onofrio F. Hypertrophy of the masseter: a rare case associated with hypertrophic cardiomyopathy. Minerva Stomatologica 1992;41(11):535-42.

\section{Harriman 1996}

Harriman DG. The histochemistry of reactive masticatory muscle hypertrophy. Muscle and Nerve 1996;19:1447-56.

\section{Higgins 2011}

Higgins JPT, Green S (editors). Cochrane Handbook for Systematic Reviews of Interventions Version 5.1.0 [updated March 2011]. The Cochrane Collaboration, 2011. Available from www.cochrane-handbook.org.

\section{Jin Park 2007}

Jin Park Y, Woo Jo Y, Bang SI, Kim HJ, Lim SY, Mun GH, et al. Radiofrequency volumetric reduction for masseteric hypertrophy. Aesthetic Plastic Surgery 2007;31(1):42-52.

\section{Kim 2000}

Kim HJ, Jeon BS, Lee KW. Hemimasticatory spasm associated with localized scleroderma and facial hemiatrophy. Archives of Neurology 2000;57(4):576-80.

\section{Kitagawa 2000}

Kitagawa Y, Hashimoto K, Kuriyama M. Hypertrophic branchial myopathy with uniform predominance of type 1 fibres. Case report. Scandinavian Journal of Plastic and Reconstructive Surgery and Hand Surgery 2000;34(4):391-6.

\section{Legg 1880}

Legg JW. Enlargement of the temporal and masseter muscles on both sides. Transactions of the Pathological Society of London 1880;31:361-6.

\section{Newton 1999}

Newton JP, Cowpe JG, McClure IJ, Delday MI, Maltin CA Masseteric hypertrophy?: preliminary report. British Journal of Oral and Maxillofacial Surgery 1999;37(5):405-8.

\section{CHARACTERISTICS OF STUDIES}

Characteristics of excluded studies [ordered by study ID]

\section{Nishida 1995}

Nishida M, lizuka T. Intraoral removal of the enlarged mandibular angle associated with masseteric hypertrophy. Journal of Oral Maxillofacial Surgery 1995;53(12):1476-9.

\section{Papapetropoulos 2006}

Papapetropoulos S, Singer C. Eating dysfunction associated with oromandibular dystonia: clinical characteristics and treatment considerations. Head and Face Medicine 2006;7(2):47.

\section{RevMan 2012 [Computer program]}

The Nordic Cochrane Centre, The Cochrane Collaboration. Review Manager (RevMan). Version 5.2. Copenhagen: The Nordic Cochrane Centre, The Cochrane Collaboration, 2012.

\section{Sannomya 2006}

Sannomya EK, Gonçalves M, Cavalcanti MP. Masseter muscle hypertrophy: case report. Brazilian Dental Journal 2006;17(4):347-50.

\section{Satoh 2001}

Satoh K, Yamaguchi T, Komatsu K, Inoue N, Minowa K, Kanayama T, et al. Analyses of muscular activity, energy metabolism, and muscle fiber type composition in a patient with bilateral masseteric hypertrophy. Cranio 2001;19(4):294-301.

\section{Serrat 1998}

Serrat A, Garcia-Cantera JM, Redondo LM. Isolated unilateral temporalis muscle hypertrophy. A case report. International Journal of Oral Maxillofacial Surgery 1998;27(2):92-3.

\section{Skoura 2001}

Skoura C, Mourouzis C, Saranteas T, Chatzigianni E, Tesseromatis $\mathrm{C}$. Masseteric hypertrophy associated with administration of anabolic steroids and unilateral mastication: a case report. Oral Surgery, Oral Medicine, Oral Pathology, Oral Radiology and Endodontics 2001;92(5):515-8.

\section{Wilson 1990}

Wilson PS, Brown AM. Unilateral temporalis muscle hypertrophy: Case report. International Journal of Oral Maxillofacial Surgery 1990;19(5):287-8.

\section{References to other published versions of this review Al-Muharraqi 2009}

Al-Muharraqi MA, Fedorowicz Z, Al Bareeq J, Al Bareeq R, Nasser M. Botulinum toxin for masseter hypertrophy. Cochrane Database of Systematic Reviews 2009, Issue 1. [DOI: 10.1002/14651858.CD007510.pub2] 


\begin{tabular}{|c|c|}
\hline Study & Reason for exclusion \\
\hline Ahn 2007 & Non relevant study design \\
\hline Bhogal 2006 & Review with no additional studies with the relevant study design \\
\hline Castro 2005 & Non relevant study design \\
\hline Chikani 2003 & Characteristics of participants do not match our inclusion criteria \\
\hline Choe 2005 & Non relevant study design \\
\hline Collier 2009 & No control group \\
\hline Hui 2002 & Letter, no relevant studies \\
\hline Işeri 2004 & Case study with no additional references to studies with the relevant study design \\
\hline $\operatorname{Jin} 2010$ & Not placebo-controlled \\
\hline Kane 2007 & Comment on Yu 2007 \\
\hline Kaynar 1997 & No control group \\
\hline Kim 2003 & Single intervention no control group \\
\hline Lee 2007 & Non relevant study design \\
\hline Mischkowski 2005 & Case series in German (translated by Mona Nasser) \\
\hline Moore 1994 & $\mathrm{~N}$ of 1 study and results not generalisable \\
\hline Niamtu 1999 & Review, no additional studies with the relevant study design \\
\hline Park 2003 & No control group \\
\hline Rogers 1995 & $\begin{array}{l}\text { Letter commenting on: Rocco R et al. Masseter hypertrophy: report of case and literature review. } \\
\text { Journal of Oral and Maxillofacial Surgery } 1994 \text { 52(11):1199-202. No further studies matching the in- } \\
\text { clusion criteria }\end{array}$ \\
\hline Smyth 1994 & Non relevant study design \\
\hline To 2001 & Non relevant study design \\
\hline von Lindern 2001 & Non relevant study design \\
\hline von Lindern 2003 & No participants with masseter hypertrophy \\
\hline Yu 2007 & Non relevant study design \\
\hline
\end{tabular}




\section{AP PE N DICES}

\section{Appendix 1. MEDLINE (via PubMed) search strategy}

("masseter"[all fields] OR masseter*[all fields] OR (("masseter"[all fields] OR masseter^[all fields] OR "Masseter muscle"[mesh] OR "Mandible"[mesh] OR "Facial Muscles"[mesh] OR masset*[all fields] OR "Masseter"[all fields] OR (("Face"[mesh] OR "face"[all fields] OR "facial"[all fields]) AND ("Muscles"[mesh] OR "muscle"[all fields] OR "muscles"[all fields] OR Muscle*[all fields])) OR (Masseter* AND Muscle $^{\star}$ ) OR Mandibl*[all fields] OR Myofac*[all fields]) AND ("Hypertrophy"[mesh] OR hypertrop*[all fields] OR enlarge*[all fields] OR thick*[all fields])) OR "Myofascial Pain Syndromes"[Mesh]) AND ("Botulinum Toxins"[mesh] OR (Botul*[all fields] AND toxi*[all fields]) OR (Botul*[all fields] AND inject*[all fields]) OR "Botulinum"[all fields] OR Botulin*[all fields] OR "botox"[all fields] OR botox*[all fields] OR "onabotulinumtoxinA"[Supplementary Concept] OR (("neurotoxins"[MeSH Terms] OR "neurotoxins"[all fields] OR "neurotoxin"[all fields] OR "neurotoxins"[Pharmacological Action] OR neurotoxin*[all fields]) AND ("Clostridium"[mesh] OR "clostridium"[all fields] OR Clostrid*[all fields])) OR "botulinal toxin"[all fields] OR "botulinium" OR botulinum OR "botulism toxin"[all fields] OR "botulism toxins" OR "abobotulinumtoxin"[all fields] OR "azzalure"[all fields] OR "bocouture"[all fields] OR "BoNT A"[all fields] OR "BoNT serotype A"[all fields] OR "botox" OR botox* OR "botulin A"[all fields] OR "botulin toxin a"[all fields] OR "BTXA"[all fields] OR "dysport"[all fields] OR evabotulinum*[all fields] OR "incobotulinum"[all fields] OR incobotulin*[all fields] OR "nt 201"[all fields] OR "nt201"[all fields] OR "oculinum"[all fields] OR "onabotulinum"[all fields] OR onabotulin*[all fields] OR "prosigne"[all fields] OR "reloxin"[all fields] OR "vistabel"[all fields] OR "xeomin"[all fields] OR "BoNT B"[all fields] OR "myobloc"[all fields] OR "myoblock"[all fields] OR "neurobloc"[all fields] OR rimabotulin*[all fields] OR "BoNT F"[all fields] OR "BoNT serotype F"[all fields] OR "BoNTF"[all fields] OR "exoenzyme C3"[all fields] OR "bacterial toxin C3"[all fields] OR "dyslor"[all fields] OR "evabotulinum"[all fields] OR "onaclostox"[all fields] OR "purtox"[all fields] OR "vistabex"[all fields] OR "rimabotulinum"[all fields])

((((Masseter muscle [mh]) OR Mandible [mh] OR (Facial Muscles [mh]) OR masset $\left.{ }^{\star}\right)$ OR (((Face OR Facial) AND Muscle $\left.{ }^{\star}\right)$ OR (Masseter ${ }^{\star}$ AND Muscle*) OR Mandibl* OR (Myofac $\left.\left.{ }^{\star}\right)\right)$ ) AND (Hypertrophy [mh] OR hypertrop* OR enlarge* OR thick*) OR (Myofascial Pain Syndromes [Mesh])) AND ((Botulinum Toxins $[\mathrm{mh}])$ OR $\left(\right.$ Botul $^{\star}$ AND toxi $\left.{ }^{\star}\right)$ OR $\left(\right.$ Botul $^{\star}$ AND inject $\left.\left.{ }^{\star}\right)\right)$

\section{Appendix 2. EMBASE (via embase.com) search strategy}

(masseter muscle/ OR masseter ${ }^{\star}$.af OR (myofascial pain/ AND hypertrop*.af) OR ((exp hypertrophy/ OR hypertrop*.af OR enlarge ${ }^{\star}$.af OR thick ${ }^{\star}$.af ) AND ((exp face/ OR facial.af OR face.af) AND muscle*.af) OR (masseter* AND muscle*).af OR mandibl*.af OR myofac*.af)) AND (botulinum toxin/ OR botulinum toxin A/ OR botulinum toxin B/ OR botulinum toxin E/ OR botulinum toxin F/ OR ("botulinal toxin" OR "botulinium" OR botulinum OR "botulism toxin" OR "botulism toxins" OR "abobotulinumtoxin" OR "azzalure" OR "bocouture" OR "BoNT A" OR "BoNT serotype A" OR "botox" OR botox* OR "botulin A" OR "botulin toxin a" OR "BTXA" OR "dyslor" OR "dysport" OR "evabotulinum" OR evabotulinum* OR "incobotulinum" OR incobotulin* OR "nt 201" OR "nt201" OR "oculinum" OR "onabotulinum" OR onabotulin* OR "onaclostox" OR "prosigne" OR "purtox" OR "reloxin" OR "vistabel" OR "vistabex" OR "xeomin" OR "BoNT B" OR "myobloc" OR "myoblock" OR "neurobloc" OR "rimabotulinum" OR rimabotulin* OR "BONT F" OR "BoNT serotype F" OR "BoNTF" OR "exoenzyme C3" OR "bacterial toxin C3").af OR (botul* AND (toxi ${ }^{\star}$ OR inject*)).af)

\section{Appendix 3. Web of Science search strategy}

TS $=\left(\left(^{\star}\right.\right.$ masseter ${ }^{\star}$ OR ("myofascial pain" AND hypertrop*) OR ((hypertrop* OR enlarge ${ }^{\star}$ OR thick $\left.{ }^{\star}\right)$ AND ((facial OR face) AND muscle $\left.{ }^{\star}\right)$ OR (masseter* AND muscle*) OR mandibl* OR myofac*)) AND ("botulin* OR botulinum toxin OR botulinum toxin A OR botulinum toxin B OR botulinum toxin $\mathrm{E}$ OR botulinum toxin $\mathrm{F} O \mathrm{OR}$ "botulinal toxin" OR "botulinium" OR botulinum OR "botulism toxin" OR "botulism toxins" OR "abobotulinumtoxin" OR "azzalure" OR "bocouture" OR "BoNT A" OR "BoNT serotype A" OR "botox" OR botox* OR "botulin A" OR "botulin toxin a" OR "BTXA" OR "dyslor" OR "dysport" OR "evabotulinum" OR evabotulinum* OR "incobotulinum" OR incobotulin^ OR "nt 201" OR "nt201" OR "oculinum" OR "onabotulinum" OR onabotulin* OR "onaclostox" OR "prosigne" OR "purtox" OR "reloxin" OR "vistabel" OR "vistabex" OR "xeomin" OR "BoNT B" OR "myobloc" OR "myoblock" OR "neurobloc" OR "rimabotulinum" OR rimabotulin^ OR "BoNT F" OR "BoNT serotype F" OR "BoNTF" OR "exoenzyme C3" OR "bacterial toxin C3" OR (botul* AND (toxi OR inject*))))

Plus citations to Cochrane Review and citations from Cochrane Review.

\section{Appendix 4. CENTRAL search strategy}

((masseter ${ }^{\star}$ OR ("myofascial pain" AND hypertrop $\left.{ }^{\star}\right)$ OR ((hypertrop ${ }^{\star}$ OR enlarge OR thick $\left.^{\star}\right)$ AND ((facial OR face) AND muscle*) OR (masseter* AND muscle*) OR mandibl* OR myofac*)) AND (botulin* OR botulinum toxin OR botulinum toxin A OR botulinum toxin B OR botulinum toxin $\mathrm{E}$ OR botulinum toxin F OR "botulinal toxin" OR "botulinium" OR botulinum OR "botulism toxin" OR "botulism toxins" OR "abobotulinumtoxin" OR "azzalure" OR "bocouture" OR "BoNT A" OR "BoNT serotype A" OR "botox" OR botox* OR "botulin A" OR "botulin toxin a" OR "BTXA" OR "dyslor" OR "dysport" OR "evabotulinum" OR evabotulinum* OR "incobotulinum" OR incobotulin* OR "nt 201" OR "nt201" OR "oculinum" OR "onabotulinum" OR onabotulin* OR "onaclostox" OR "prosigne" OR "purtox" OR "reloxin" OR "vistabel" OR "vistabex" OR "xeomin" OR "BoNT B" OR "myobloc" OR "myoblock" OR "neurobloc" OR "rimabotulinum" OR rimabotulin* OR "BoNT F" OR "BoNT serotype F" OR "BoNTF" OR "exoenzyme C3" OR "bacterial toxin C3" OR (botul* AND (toxi* OR inject*))))

\section{Appendix 5. CINAHL (via EbscoHost) search strategy}

In fields: ti/ab/kw 
((masseter ${ }^{\star}$ OR ("myofascial pain" AND hypertrop*) OR ((hypertrop* OR enlarge* OR thick*) AND ((facial OR face) AND muscle*) OR (masseter* AND muscle*) OR mandibl* OR myofac*)) AND (botulin* OR botulinum toxin OR botulinum toxin A OR botulinum toxin B OR botulinum toxin E OR botulinum toxin F OR "botulinal toxin" OR "botulinium" OR botulinum OR "botulism toxin" OR "botulism toxins" OR "abobotulinumtoxin" OR "azzalure" OR "bocouture" OR "BoNT A" OR "BoNT serotype A" OR "botox" OR botox* OR "botulin A" OR "botulin toxin a" OR "BTXA" OR "dyslor" OR "dysport" OR "evabotulinum" OR evabotulinum OR "incobotulinum" OR incobotulin* OR "nt 201" OR "nt201" OR "oculinum" OR "onabotulinum" OR onabotulin* OR "onaclostox" OR "prosigne" OR "purtox" OR "reloxin" OR "vistabel" OR "vistabex" OR "xeomin" OR "BoNT B" OR "myobloc" OR "myoblock" OR "neurobloc" OR "rimabotulinum" OR rimabotulin* OR "BoNT F" OR "BoNT serotype F" OR "BoNTF" OR "exoenzyme C3" OR "bacterial toxin C3" OR (botul* AND (toxi* OR inject*))))

\section{Appendix 6. Academic Search Premier (via EbscoHost) search strategy}

In fields: ti/ab/kw/su

((masseter* OR ("myofascial pain" AND hypertrop*) OR ((hypertrop* OR enlarge* OR thick ${ }^{\star}$ ) AND ((facial OR face) AND muscle $)$ OR (masseter ${ }^{\star}$ AND muscle ${ }^{\star}$ ) OR mandibl ${ }^{\star}$ OR myofac $\left.{ }^{\star}\right)$ AND (botulin* OR botulinum toxin OR botulinum toxin A OR botulinum toxin B OR botulinum toxin E OR botulinum toxin F OR "botulinal toxin" OR "botulinium" OR botulinum OR "botulism toxin" OR "botulism toxins" OR "abobotulinumtoxin" OR "azzalure" OR "bocouture" OR "BoNT A" OR "BoNT serotype A" OR "botox" OR botox* OR "botulin A" OR "botulin toxin a" OR "BTXA" OR "dyslor" OR "dysport" OR "evabotulinum" OR evabotulinum* OR "incobotulinum" OR incobotulin* OR "nt 201" OR "nt201" OR "oculinum" OR "onabotulinum" OR onabotulin* OR "onaclostox" OR "prosigne" OR "purtox" OR "reloxin" OR "vistabel" OR "vistabex" OR "xeomin" OR "BoNT B" OR "myobloc" OR "myoblock" OR "neurobloc" OR "rimabotulinum" OR rimabotulin* OR "BoNT F" OR "BoNT serotype F" OR "BoNTF" OR "exoenzyme C3" OR "bacterial toxin C3" OR (botul* AND (toxi OR inject*))))

\section{Appendix 7. ScienceDirect search strategy}

TITLE-ABSTR-KEY(((masseter* OR ("myofascial pain" AND hypertrop*) OR ((hypertrop* OR enlarge* OR thick*) AND ((facial OR face) AND muscle ${ }^{\star}$ ) OR (masseter ${ }^{\star}$ AND muscle ${ }^{\star}$ ) OR mandibl ${ }^{\star}$ OR myofac $\left.{ }^{\star}\right)$ ) AND (botulin ${ }^{\star}$ OR botulinum toxin OR botulinum toxin A OR botulinum toxin B OR botulinum toxin E OR botulinum toxin F OR "botulinal toxin" OR "botulinium" OR botulinum OR "botulism toxin" OR "botulism toxins" OR "abobotulinumtoxin" OR "azzalure" OR "bocouture" OR "BoNT A" OR "BoNT serotype A" OR "botox" OR botox* OR "botulin A" OR "botulin toxin a" OR "BTXA" OR "dyslor" OR "dysport" OR "evabotulinum" OR evabotulinum* OR "incobotulinum" OR incobotulin* OR "nt 201" OR "nt201" OR "oculinum" OR "onabotulinum" OR onabotulin^ OR "onaclostox" OR "prosigne" OR "purtox" OR "reloxin" OR "vistabel" OR "vistabex" OR "xeomin" OR "BoNT B" OR "myobloc" OR "myoblock" OR "neurobloc" OR "rimabotulinum" OR rimabotulin* OR "BoNT F" OR "BoNT serotype F" OR "BoNTF" OR "exoenzyme C3" OR "bacterial toxin C3" OR (botul* AND (toxi OR inject*)))))

\section{Appendix 8. LILACS (via Bireme) search strategy}

masseter AND botulinum

\section{Appendix 9. PubMed Central search strategy}

masseter ${ }^{\star}$ AND (hypertrop* OR enlarge ${ }^{\star}$ OR thick ${ }^{\star}$ ) AND (botulin* OR botulinum toxin OR botulinum toxin A OR botulinum toxin B OR botulinum toxin E OR botulinum toxin F OR "botulinal toxin" OR "botulinium" OR botulinum OR "botulism toxin" OR "botulism toxins" OR "abobotulinumtoxin" OR "azzalure" OR "bocouture" OR "BoNT A" OR "BoNT serotype A" OR "botox" OR botox* OR "botulin A" OR "botulin toxin a" OR "BTXA" OR "dyslor" OR "dysport" OR "evabotulinum" OR evabotulinum* OR "incobotulinum" OR incobotulin* OR "nt 201" OR "nt201" OR "oculinum" OR "onabotulinum" OR onabotulin^ OR "onaclostox" OR "prosigne" OR "purtox" OR "reloxin" OR "vistabel" OR "vistabex" OR "xeomin" OR "BoNT B" OR "myobloc" OR "myoblock" OR "neurobloc" OR "rimabotulinum" OR rimabotulin* OR "BoNT F" OR "BoNT serotype F" OR "BoNTF" OR "exoenzyme C3" OR "bacterial toxin C3") AND (randomized OR randomised OR random OR RCT OR RCTS OR trial OR trials OR placebo OR placebos OR compared OR comparison OR versus)

\section{Appendix 10. Google Scholar search strategy}

allintitle:masseter allintitle:botulinum

Plus: citations to Cochrane Review

WHAT'S NEW

\begin{tabular}{lll}
\hline Date & Event & Description \\
\hline 26 April 2013 & $\begin{array}{l}\text { New citation required but conclusions } \\
\text { have not changed }\end{array}$ & New authors added \\
\hline 26 April 2013 & New search has been performed & $\begin{array}{l}\text { Search strategy updated } \\
\text { Searches updated }\end{array}$ \\
\hline
\end{tabular}




\section{CONTRIBUTIONS OF AUTHORS}

Zbys Fedorowicz (ZF), Esther van Zuuren (EvZ), Jan Schoones (JS) were responsible for:

Conducting all the searches for this update (JS)

- organising the retrieval of papers;

- writing to authors of papers for additional information;

- screening search results;

- screening retrieved papers against inclusion criteria;

- appraising the quality of papers;

- data collection for the review;

- extracting data from papers; and

- obtaining and screening data on unpublished studies.

ZF and EvZ were responsible for entering any extracted data into RevMan.

ZF and EvZ were responsible for the analysis and interpretation of data.

ZF was responsible for:

- designing the review;

- co-ordinating the review;and

- data management for the review.

All review authors contributed to writing the review.

ZF conceived the idea for the review and is the guarantor for the review.

\section{DECLARATIONS OF INTEREST}

There are no financial conflicts of interest and the authors declare that they do not have any associations with any parties who may have vested interests in the results of this review.

\section{SOURCES OF SUPPORT}

\section{Internal sources}

- No sources of support, Netherlands.

\section{External sources}

- No sources of support, Netherlands.

\section{DIFFERENCES BETWEEN PROTOCOLANDREVIEW}

We added Adverse outcomes under 'Types of outcome measures'.

\section{INDEX TERMS}

\section{Medical Subject Headings (MeSH)}

Botulinum Toxins, Type A [*therapeutic use]; Hypertrophy [drug therapy]; Injections, Intramuscular; Masseter Muscle [ ${ }^{\star}$ pathology]; Neuromuscular Agents [ ${ }^{\star}$ therapeutic use]

\section{MeSH check words}

Humans 